FDP und Grüne sind ein gutes Beispiel dafür, welche Variationen hinsichtlich der Repräsentation und der Entscheidungsfindung auf Parteitagen trotz der vereinheitlichenden Wirkung von Gesetzgebung, Rechtsprechung und den Erfordernissen der modernen (Medien-) Demokratie möglich sind. Dies betrifft zum einen Unterschiede innerhalb einer Partei, wie die Grünen zeigen, aber auch zwischen den Parteien, wo wiederum die durch Geschichte, unterschiedlichen Status und Größe bedingten Diskrepanzen zwischen den kleinen beziehungsweise mittleren Parteien einerseits und den großen Volksparteien andererseits sichtbar werden.

Diese Ergebnisse sind ein guter Ausgangspunkt für weitere Forschungen: So ließe sich genauer untersuchen, inwieweit die organisatorisch-kulturellen Unterschiede auf unterschiedliche ideologische Standpunkte, unterschiedlichen Entstehungshintergrund und -zeitpunkt oder eine Kombination hiervon zurückzuführen sind. Auch eine genauere Analyse von wichtigen Aspekten der Entscheidungskultur wie Verhandlungen und informellen Gesprächen wäre aufschlussreich. Zudem bleibt die weitere Entwicklung der Piratenpartei abzuwarten. Im Falle einer dauerhaften Etablierung wird es hochinteressant sein, zu sehen, wie und in welchem Umfang dieser neue Akteur seinen innovativen organisatorischen Ansatz, dessen Forderung nach Transparenz frappierend an die frühen Grünen erinnert, beibehalten (können) wird. Die ersten parteiinternen Diskussionen rund um das Mandatsverständnis und die Bezahlung von Vorständen lassen auf einen den Grünen nicht unähnlichen Anpassungsdruck schließen.

\title{
Der Mundatwald bleibt deutsch! Wie das deutsch-französische Grenzabkommen vom 31. Juli 1962 am Auswärtigen Ausschuss des Bundestages scheiterte*
}

\author{
Volker Pilz
}

Der bislang einzige Fall, in dem die Zustimmung zu einem von der Bundesregierung ausgehandelten völkerrechtlichen Vertrag vom Bundestag verweigert wurde, ist das deutschfranzösische Grenzabkommen vom 31. Juli 1962. Diesem Abkommen zufolge sollte ein etwa sieben Quadratkilometer großes Gebiet in der Südpfalz, der Mundatwald, an Frankreich abgetreten werden. Der Auswärtige Ausschuss des Bundestages verhinderte die Ratifizierung des Vertrages, indem er es nach umfangreichen Beratungen unterließ, das Zustimmungsgesetz dem Plenum zur endgültigen Beschlussfassung vorzulegen. Dieser bislang einzige Fall verweigerter parlamentarischer Zustimmung bei der Ratifizierung völkerrechtlicher Verträge gemäß Art. 59 Abs. 2 Satz 1 GG soll im Folgenden näher beleuchtet werden.

* Der Autor dankt Edouard Michel, Ständiger Vertreter der französischen Nationalversammlung bei der Europäischen Union, für seine Unterstützung bei der Dokumentenrecherche. 


\section{Vorgeschichte und Inhalt des deutsch-französischen Grenzabkommens vom 31. Juli 1962}

Der südpfälzische Mundatwald war nach dem Zweiten Weltkrieg von der französischen Besatzungsmacht unter eigene Verwaltungshoheit gestellt worden. ${ }^{1}$ Rechtsgrundlage war die Verordnung Nr. 212 über Grenzberichtigungen vom 23. April 1949, in der der französische Oberbefehlshaber in Deutschland, General Pierre Koenig, verschiedene vorläufige Änderungen der deutschen Westgrenze anordnete. ${ }^{2}$ Formell wurde dies damit begründet, dass durch die im Gebiet des Mundatwaldes gelegenen Quellen die Trinkwasserversorgung der französischen Stadt Weißenburg im Elsass sichergestellt werden sollte. ${ }^{3}$

Am 23. April 1949 markierten französische Forst- und Vermessungsbeamte den künftigen Grenzverlauf. Dabei wurde ein Keil von etwa drei Kilometer Tiefe in das deutsche Gebiet hinein getrieben, obwohl die Quelle, die zur Wasserversorgung der Stadt Weißenburg beansprucht wurde, nur $600 \mathrm{~m}$ von der alten Reichsgrenze entfernt lag. Bei dieser Gelegenheit kam auch die in dem Gebiet gelegene Siedlung St. Germanshof unter vorläufige französische Verwaltung. Auf Protest des rheinland-pfälzischen Ministerpräsidenten Peter Altmeier (CDU) wurde das Gebiet von St. Germanshof am 9. September 1949 zwar wieder zurückgegeben. Dafür trieben die Franzosen den vorerwähnten Keil aber um weitere zwei Kilometer gen Norden, so dass er den höchsten Berg der Südpfalz, die „Hohe Derst“, sowie die Guttenberger Schlossruine mit umfasste und nunmehr eine Gesamttiefe von etwa 5,2 Kilometer hatte. ${ }^{4}$ Das 6,9 Quadratkilometer große Gebiet des Mundatwaldes stand damit unter alleiniger französischer Verwaltung.

Bei diesem Status quo blieb es lange Jahre: Zum einen, weil nach der seit Oktober 1945 in Kraft getretenen und für Frankreich verbindlichen UN-Charta die Besatzungsmacht an dem politischen Status eines besetzten Gebietes nichts ändern darf ${ }^{5}$, zum anderen, weil die Alliierten selbst nach der Kapitulation des Deutschen Reiches die Absicht geäußert hatten,

1 Zur wechselhaften territorialen Geschichte des Mundatwaldes siehe Ansbert Baumann, Konfliktlinie oder Freundschaftsband? Verdun, Mundatwald und Schengen - die Entwicklung der deutschfranzösischen Grenze, in: Revue d'Allemagne et de pays de langue allemande, 41. Jg. (2009), H. 4, S. 619 - 633, S. 625 ff. sowie Heidi Dünisch, Der Mundatwald - Zur Bereinigung letzter Kriegsfolgenprobleme zwischen Deutschland und Frankreich, Frankfurt am Main u.a. 1989 (zugleich Dissertation an der Universität Würzburg), S. 5 f., S. 13 ff.

2 Die Verordnung erfolgte auf Grundlage der Beschlüsse der Londoner Sechs-Mächte-Konferenz von 1948. Für den genauen Wortlaut siehe Journal officiel du Commandement en Chef Français en Allemagne (Amtsblatt des französischen Oberkommandos in Deutschland), Nr. 262, S. $1969 \mathrm{f}$. Abgedruckt in Dietrich Rauschning, Die Gesamtverfassung Deutschlands - Nationale und internationale Texte zur Rechtslage Deutschlands, Frankfurt am Main 1962, Bd. I, Nr. 31 III, S. $696 \mathrm{f}$.

3 So der Wortlaut des Artikels 1 Ziff. 4 Satz 1 der Verordnung Nr. 212 vom 23. April 1949: „Zwischen Frankreich und Deutschland (Staat Rheinland-Pfalz) werden in dem Forst von Mundat das Tal des Burbachs und die Quellen, die die Stadt Wissembourg mit Trinkwasser versorgen, dem französischen Gebiet einverleibt."

4 Vgl. Landeshauptarchiv Koblenz, 860, 19. März 2001, Nr. 1012; Landesarchiv Speyer, H 500 Nr. 1955. Siehe dazu auch Dieter Schröder, Ein und kein Federstrich machen den Mundatwald französisch, in: Rheinpfalz Ludwigshafen vom 8. Dezember 1961; Karl-Heinz Baum, Freundschaft in der Schwebe, in: Die Zeit vom 7. März 1975, S. 11.

5 Völkerrechtliches Annexionsverbot gemäß Art. 2 Ziff. 4 UN-Charta. 
jedenfalls zunächst die Grenzen des Deutschen Reiches vom 31. Dezember 1937 aufrecht zu erhalten. ${ }^{6}$ Die Gebietskarten wiesen den Mundatwald seither als „deutsches Gebiet unter vorläufiger französischer Verwaltung" aus. ${ }^{7}$

Da eine Regelung der offenen Grenzfrage im Rahmen eines Friedensvertrages auf sich warten ließ und auch in den Verträgen für Saar, Mosel und Oberrhein vom 27. Oktober 1956 keine Klärung der Mundatwaldfrage erfolgte, kam den Franzosen ein Vorstoß der Mainzer Staatskanzlei im Jahre 1962 gerade recht: Rheinland-Pfalz wollte im Elsass gelegenen und von den Franzosen im Oktober 1944 sequestrierten grenznahen privaten Grundbesitz an Äckern, Wiesen und Weinbergen zurückerhalten. ${ }^{8}$ Im Gegenzug forderten die Franzosen die endgültige Abtretung des Mundatwaldes an Frankreich und boten als Draufgabe und gleichermaßen Bonbon die Rückübertragung der ebenfalls sequestrierten Christuskirche in Paris an. ${ }^{9}$

Dieser Tausch wurde den Verhandlungen über das deutsch-französische Grenzabkommen von 1962 zugrunde gelegt. Die französischen Diplomaten verhandelten erfolgreich, so dass das Abkommen schließlich ganz in ihrem Sinne die endgültige Abtretung des Mundatwaldes an Frankreich vorsah. ${ }^{10}$ Im Gegenzug wurde die Rückgabe des in Frankreich gelegenen sequestrierten grenznahen privaten Grundbesitzes (Sequesterland) an die deutschen Eigentümer und die Rückübertragung des Eigentums an der Christuskirche in Paris an die dortige evangelische Gemeinde vereinbart. ${ }^{11}$ Mit diesem Inhalt wurde das Abkommen schließlich am 31. Juli 1962 von beiden Seiten in Paris paraphiert.

\section{Ratifizierungsverfahren in Frankreich}

Vom französischen Parlament wurde das deutsch-französische Grenzabkommen Anfang Juni 1963 gebilligt $^{12}$ und am 8. August 1963 im „Journal Officiel de la République Française“ veröffentlicht. ${ }^{13}$ Damit besaß es für die französische Seite Rechtskraft.

6 Vgl. Fritz Münch, Zum Saarvertrag vom 27. Oktober 1956, in: ZaöRV, 18. Jg. (1957/58), S. 1 -60, S. 2.

7 Günter Baumann / Hans Wadlinger, Der Mundatwald, ein deutsch-französisches Grenzproblem wird bereinigt, in: Nachrichtenblatt der Vermessungs- und Katasterverwaltung Rheinland-Pfalz, 29. Jg. (1986), H. 1, S. 2 - 13.

8 Es handelte sich um Grundstücke in der Größenordnung von circa 600 Hektar, die entlang der deutsch-französischen Grenze auf französischem Territorium lagen.

9 Vgl. Hans-Joachim Noack, Tausche Pariser Kirche gegen deutschen Wald, in: Die Zeit vom 10. März 1967, S. 13.

10 Vgl. „Die deutsch-französische Freundschaft hört im Mundatwald auf: Ein Paradebeispiel für die Verhandlungs-,Kunst’ der Franzosen“, in: Bremer Nachrichten vom 15. April 1966.

11 Zum Vertragstext siehe BT-Drs. 5/405.

12 Siehe dazu „Deutsch-französische Grenzkorrektur gebilligt“ in: Die Welt vom 7. Juni 1963; „L'Assemblée nationale approuve une légère rectification de la frontière franco-allemande“ in: Le Monde vom 8. Juni 1963.

13 Journal Officiel de la République Française, 8. August 1963, S. 7356 ff. 


\section{Ratifizierungsverfahren in Deutschland}

In Deutschland wurde das Ratifikationsgesetz zu dem Abkommen im Mai 1963 dem Bundesrat zugeleitet. ${ }^{14}$ Im Vorfeld seiner Beratungen war der Druck auf alle Parteien in Rheinland-Pfalz allerdings so groß geworden, dass sämtliche Fraktionen des Landtags von Rheinland-Pfalz die Landesregierung drängten, im Bundesrat gegen das Abkommen zu stimmen. Ministerpräsident Peter Altmeier sah sich daher veranlasst, dem Abkommen in der 259. Sitzung des Bundesrates am 21. Juni 1963 die Zustimmung zu verweigern. ${ }^{15}$ Diesem ablehnenden Votum schloss sich auch das Land Hessen an. ${ }^{16}$ Der Bundesrat stimmte dem Abkommen zwar mehrheitlich zu und erklärte das Ratifizierungsgesetz wegen Art. 29 Abs. 7 GG zugleich für zustimmungsbedürftig. ${ }^{17}$ Aufgrund des Widerstandes der Bundesländer Rheinland-Pfalz und Hessen, aber auch wegen interner Widerstände ${ }^{18}$ brachte die Bundesregierung das Ratifikationsgesetz zu dem Abkommen in der damals laufenden 4. Wahlperiode jedoch nicht mehr in den Bundestag ein.

\subsection{Eingabe des Notars Karl Bertzel aus Zweibrücken}

Gleichwohl befasste sich der Auswärtige Ausschuss des Bundestages aufgrund einer Eingabe des Notars Karl Bertzel aus Zweibrücken bereits in der 4. Wahlperiode mit dem Abkommen. ${ }^{19}$ Bertzel, gewissermaßen der spiritus rector der Südpfälzer Protestbewegung, hatte sich mit seiner Eingabe vom 16. Oktober 1963 gegen die in Artikel 8 des Grenzabkommens vorgesehene Abtretung des Mundatwaldes an Frankreich gewendet. ${ }^{20}$ Auf seiner 40. Sitzung am 7. November 1963 ließ sich der Auswärtige Ausschuss durch das Auswärtige Amt über den Stand des Ratifizierungsverfahrens sowie die Widerstände in Rheinland-Pfalz gegen die

14 BR-Drs. 235/63.

15 Vgl. „Altmeier stimmt Abkommen nicht zu“, in: Saarbrücker Landeszeitung vom 22. Juni 1963; „Deutsch-französisches Grenzabkommen im Bundesrat“ und „Der Mundatwald“, in: FAZ vom 22. Juni 1963.

16 Verhandlungen des Bundesrates 1963, Stenographischer Bericht der 259. Sitzung vom 21. Juni 1963 , S. $136 \mathrm{f}$.

17 Ebenda, S. 137.

18 Als das Abkommen am 16. Mai 1963 im Bundeskabinett behandelt wurde, stimmte der Bundesminister für Vertriebene, Flüchtlinge und Kriegsgeschädigte Wolfgang Mischnick (FDP) gegen den Entwurf, der Bundesjustizminister Ewald Bucher (FDP) enthielt sich der Stimme, und selbst der Bundesaußenminister Gerhard Schröder (CDU) stellte fest, dass die Frage, „ob es sich bei der Festlegung der Grenze im Gebiet des Mundatwaldes lediglich um eine Grenzberichtigung oder um eine Gebietsabtretung handele, [...] zwar als streitig angesehen werden" könne, er erwarte jedoch, dass das weitere Gesetzgebungsverfahren diesbezüglich eine Klärung bringen würde. Siehe Hartmut Weber (Hrsg.), Die Kabinettsprotokolle der Bundesregierung, Bd. 16 (1963), München 2006, S. 233.

19 Vgl. Der Auswärtige Ausschuss des Deutschen Bundestages, Sitzungsprotokolle 1961-1965, Halbbd. 2: Juni 1963 bis September 1965, bearbeitet von Wolfgang Hölscher, Düsseldorf 2004, CDROM-Supplement, 40. Sitzung am 7. November 1963, S. 918 f. und 41. Sitzung am 14. November 1963, S. $926 \mathrm{ff}$.

20 Wortlaut des Artikels 8: „Um den örtlichen Bedürfnissen, insbesondere der Trinkwasserversorgung der Stadt Weißenburg, Rechnung zu tragen, erkennen die Französische Republik und die Bundesrepublik Deutschland den Grenzverlauf im Gebiet von Weißenburg als endgültig an [...]." BT-Drs. 5/405, S. 5. 
Abtretung des Mundatwaldes unterrichten. ${ }^{21}$ Der Legationsrat Erster Klasse Richard Balken (AA) teilte mit, dass nach Ansicht des Bundesrates das Zustimmungsgesetz zu dem Abkommen gemäß Art. 29 Abs. 7 GG der Zustimmung der Länderkammer bedürfe. In der Regierungsvorlage sei eine solche Zustimmung bislang aber nicht vorgesehen, weshalb eine Einfügung der Worte „mit Zustimmung des Bundesrates“ in die Eingangsformel des Zustimmungsgesetzes erfolgen solle. Überdies sei die rheinland-pfälzische Landesregierung durch Beschluss des Landtages vom 9. Juli 1963 gebeten worden, auf eine Neuregelung mit Frankreich zu drängen. Dem Wunsch der Landesregierung entsprechend habe das Auswärtige Amt inzwischen im Rahmen der deutsch-französischen Konsultationen die Mundatwaldfrage wieder aufgenommen, bisher aber ohne Erfolg. ${ }^{22}$ Nach kurzer Diskussion beschloss der Ausschuss, die Frage in der nächsten Sitzung weiter zu erörtern. ${ }^{23}$

In der 41. Sitzung des Ausschusses am 14. November 1963 teilte der Ausschussvorsitzende Hermann Kopf (CDU) zunächst mit, er habe die Frage der Abtretung des Mundatwaldes auf die Tagesordnung gesetzt, damit im Ausschuss geklärt werden könne, ob die Bundesregierung gebeten werden solle, hierüber mit der französischen Regierung erneut zu verhandeln. ${ }^{24}$ Der zum Berichterstatter bestimmte Abgeordnete Karl Weber (CDU) berichtete sodann über die Eingabe von Karl Bertzel sowie die Hintergründe der Mundatwaldfrage. ${ }^{25}$ Rechtlich sei der Wald noch immer deutsches Territorium. Man müsse sich bei der geplanten Grenzänderung jedoch fragen, ob es sich um eine Grenzberichtigung oder eine Gebietsabtretung handele. Er neige zu der Auffassung, es sei eine Gebietsabtretung, die mit Rücksicht auf Artikel 7 Absatz 1 des Deutschlandvertrages ${ }^{26}$ aber einem Friedensvertrag mit einer gesamtdeutschen Regierung vorbehalten sei. ${ }^{27}$

Dem hielt Ministerialdirigent Hermann Meyer-Lindenberg (AA) die Auffassung des Auswärtigen Amts entgegen, dass durch Artikel 7 Absatz 1 des Deutschlandvertrages nicht jede geringfügige Grenzänderung ausgeschlossen sei. So würden insbesondere Grenzberichtigungen zulässig sein. ${ }^{28}$ Bei der in Rede stehenden Grenzänderung im Gebiet des Mundatwaldes sei jedoch zuzugeben, dass hier weder eine unzweckmäßige noch eine ungewisse Grenze bestanden habe. Es handele sich daher dort auch nicht um eine Berichtigung, sondern um eine Änderung der Grenze. Dennoch sei die Auffassung zu vertreten, dass Artikel 7 Absatz 1 des Deutschlandvertrages entsprechend seinem Sinn und Zweck derartigen kleinen territorialen Veränderungen nicht entgegenstehe, insbesondere wenn es sich um unbewohntes Gebiet handele. ${ }^{29}$ Der Preis für die in dem Grenzvertrag vereinbarte Rückgabe des im Elsass gelegenen grenznahen Gebietes (Sequesterland) sei die Anerkennung der faktisch bestehen-

21 Vgl. Sitzungsprotokolle, a.a.O. (Fn. 19), 40. Sitzung am 7. November 1963, S. 918 f.

22 Vgl. ebenda.

23 Vgl. ebenda, S. 919.

24 Vgl. Sitzungsprotokolle, a.a.O. (Fn. 19), 41. Sitzung am 14. November 1963, S. 926.

25 Vgl. ebenda, S. $926 \mathrm{f}$.

26 Wortlaut des Artikels 7 Abs. 1 des Deutschlandvertrages: „Die Unterzeichnerstaaten sind sich darüber einig, dass ein wesentliches Ziel ihrer gemeinsamen Politik eine zwischen Deutschland und seinen ehemaligen Gegnern frei vereinbarte friedensvertragliche Regelung für ganz Deutschland ist, welche die Grundlage für einen dauerhaften Frieden bilden soll. Sie sind weiterhin darüber einig, dass die endgültige Festlegung der Grenzen Deutschlands bis zu dieser Regelung aufgeschoben werden muss." Siehe BGBl. 1955 II, S. 305 - 320, S. 309.

27 Vgl. Sitzungsprotokolle, a.a.O. (Fn. 19), 41. Sitzung am 14. November 1963, S. 927.

28 Vgl. ebenda.

29 Vgl. ebenda. 
den französischen Gebietshoheit im Mundatwald. Dieses Junktim der französischen Regierung habe die deutsche Delegation vergeblich zu lösen versucht. Es seien allerdings erneute Verhandlungen im Gange, deren Ergebnis aber noch offen sei. Die Aussichten auf eine für die Bundesrepublik vorteilhaftere Lösung seien nicht besonders günstig, zumal die französische Nationalversammlung das Abkommen bereits ratifiziert habe. Trotz verständlicher Bedenken wäre die Ratifikation des Vertrages - auch in der vorliegenden Fassung erwünscht. Sicher sei, dass die Nichtratifizierung in Frankreich Verstimmung auslösen würde. ${ }^{30}$

Der Abgeordnete Herbert Wehner (SPD) erklärte, er sehe für seine Person und wohl auch für seine Fraktion keine Möglichkeit, dem Abkommen in der gegenwärtigen Form zuzustimmen. Im Rahmen der europäischen Zusammenarbeit sollte es möglich sein, die Wasserversorgung Weißenburgs auf andere Weise als durch die Abtretung deutschen Gebietes sicherzustellen. Er sei der Auffassung, die Bundesregierung solle sich in erneuten Verhandlungen um die Rückgabe des Mundatwaldes bemühen. ${ }^{31}$

In der sich anschließenden allgemeinen Aussprache wurde der Wunsch des Ausschusses nach erneuten Verhandlungen deutlich. ${ }^{32}$ Zum Abschluss der Debatte beschlossen die Ausschussmitglieder, dass der Vorsitzende das Auswärtige Amt ersuchen solle, die Verhandlungen mit der französischen Regierung erneut aufzunehmen mit dem Ziel, dass von einem Ausscheiden des Mundatwaldes aus dem deutschen Hoheitsgebiet abgesehen werden könne. ${ }^{33}$ Weitere Bemühungen der Bundesregierung zu einer Neuverhandlung des Abkommens blieben jedoch erfolglos, und weil das Ratifizierungsgesetz zu dem Abkommen noch nicht offiziell in den Bundestag eingebracht worden war, sah der Auswärtige Ausschuss in der 4. Wahlperiode keine Notwendigkeit einer weiteren Befassung.

\subsection{Beratungen über das Ratifizierungsgesetz in der 5. Wahlperiode}

Wegen der oben geschilderten schwierigen Gemengelage, aber auch wegen nach wie vor bestehender interner Widerstände ${ }^{34}$ legte die Bundesregierung das Ratifizierungsgesetz zum deutsch-französischen Grenzabkommen dem Bundestag erst in der 5. Wahlperiode am 9. März 1966 vor. ${ }^{35}$ In der 30. Sitzung des Bundestages am 16. März 1966 wurde es vom Plenum in erster Lesung ohne Aussprache an den Auswärtigen Ausschuss überwiesen. ${ }^{36} \mathrm{Mit}$

$30 \mathrm{Vgl}$. ebenda.

31 Vgl. ebenda, S. 928.

32 Vgl. ebenda.

33 Vgl. ebenda.

34 Auf der Sitzung des Bundeskabinetts am 2. Februar 1966 war die erneute Vorlage des Gesetzentwurfs an die gesetzgebenden Körperschaften auf den Widerstand dreier Ressorts gestoßen, wurde aber insbesondere mit dem Argument, dass der Vertragsschluss mit Frankreich nun endlich auch honoriert werden müsse, mehrheitlich beschlossen. Siehe Hartmut Weber (Hrsg.), Die Kabinettsprotokolle der Bundesregierung, Bd. 19 (1966), München 2009, S. $91 \mathrm{f}$.

35 BT-Drs. 5/405. Der Bundesrat hatte den Gesetzentwurf (BR-Drs. 77/66) am 4. März 1966 entsprechend der Empfehlung seines Ausschusses für Auswärtige Angelegenheiten ohne Einwendungen passieren lassen. Vgl. Verhandlungen des Bundesrates, Stenographischer Bericht der 292. Sitzung am 4. März 1966, S. 45 (B).

36 Siehe dazu das Plenarprotokoll, Verhandlungen des Deutschen Bundestages, 5. Wahlperiode, Stenographische Berichte, Band 61, 30. Sitzung am 16. März 1966, S. 1378 (D). 
dem Ratifizierungsgesetz beschäftigte sich der Auswärtige Ausschuss in seiner 36., 51., 94. und 96. Sitzung der 5. Wahlperiode. ${ }^{37}$

Die Mitglieder des Auswärtigen Ausschusses machten sich mit geradezu akribischer Genauigkeit daran, Hintergründe, Motive und Konsequenzen des Abkommens zu durchleuchten. Auf Vorschlag des Ausschussvorsitzenden Hermann Kopf wurde eine Arbeitsgruppe zur Vorberatung des deutsch-französischen Grenzabkommens zusammengestellt (so genannte „Mundatwald-Kommission") ${ }^{38}$, die auch in das Mundatwald-Gebiet reiste, um sich an Ort und Stelle zu informieren und pro und contra des Abkommens mit der Bevölkerung zu diskutieren. ${ }^{39}$

(a) Dreh- und Angelpunkt war die rechtliche Bewertung der Frage, ob es sich bei der Vereinbarung um eine schlichte Grenzberichtigung oder um eine Gebietsabtretung handele. Diese vordergründige Detailfrage war juristisch von großer Bedeutung, da in Artikel 7 des Deutschlandvertrages vom 23. Oktober 1954 festgelegt worden war, dass die endgültige Festlegung der Grenzen Deutschlands bis zur Unterzeichnung eines Friedensvertrages mit Gesamt-Deutschland aufgeschoben werde. ${ }^{40} \mathrm{Um}$ dennoch über eine gewisse Handlungsfähigkeit bei der administrativen Verwaltung der Grenzregionen verfügen zu können, hatte das Auswärtige Amt in den bereits zuvor verabschiedeten Ausgleichsabkommen mit Belgi$e^{41}$, Luxemburg ${ }^{42}$ und den Niederlanden ${ }^{43}$ eine Unterscheidung zwischen Grenzänderungen und bloßen Grenzberichtigungen vorgenommen und festgelegt, dass letztere nicht unter den Vorbehalt des Deutschlandvertrages fallen würden. Daher hatte die Bundesregierung auch im Falle des Mundatwald-Abkommens der Gesetzesvorlage eine Denkschrift angefügt, in der sie betonte, dass ,in dem Vertrag keine eigentlichen Grenzänderungen, sondern nur Grenzberichtigungen vereinbart worden " 44 seien.

Während sich das Auswärtige Amt also weiterhin auf den Standpunkt stellte, es liege lediglich eine Grenzberichtigung vor, erhob sich in der Bevölkerung der Südpfalz ein Sturm der Entrüstung. Die aufgebrachten Bürger waren der Meinung, die neue Grenzziehung sei alles andere als eine Grenzberichtigung, sondern vielmehr eine massive Erweiterung französischen Gebiets.

In einem Schreiben an den Bundeskanzler, den Bundesaußenminister, den Bundesjustizminister und an den rheinland-pfälzischen Ministerpräsidenten warnte der frühere pfälzische Oberlandesgerichtspräsident Ludwig Ritterspacher aus Neustadt an der Weinstraße die

37 Vgl. Der Auswärtige Ausschuss des Deutschen Bundestages, Sitzungsprotokolle 1965-1969, Halbbd. 1: Dezember 1965 bis Dezember 1967 und Halbbd. 2: Januar 1968 bis August 1969, bearbeitet von Joachim Wintzer, Düsseldorf 2006, CD-ROM-Supplement.

38 Ihr gehörten der Ausschussvorsitzende Hermann Kopf (CDU), der Berichterstatter zu dem Abkommen Hans-Joachim von Merkatz (CDU) sowie die Ausschussmitglieder Erhard Eppler (SPD) und Fritz-Rudolf Schultz (FDP) an. Siehe Sitzungsprotokolle, a.a.O. (Fn. 37), 12. Sitzung vom 27. Mai 1966, S. 151 und 14. Sitzung vom 22. Juni 1966, S. 170 sowie 16. Sitzung vom 30. Juni 1966, S. 195.

39 Siehe dazu den kurzen Bericht Hermann Kopfs in der 36. Sitzung des Auswärtigen Ausschusses am 21. Juni 1967, Sitzungsprotokolle, a.a.O. (Fn. 37), S. 493.

40 BGBl. 1955 II, S. 305 - 320, S. 309. Zum genauen Wortlaut siehe Fn. 26.

41 BGBl. 1958 II, S. $262-290$.

42 BGBl. 1960 II, S. $2077-2108$.

43 BGBl. 1963 II, S. $458-601$.

44 BT-Drs. 5/405, S. 14. 
Bundes- und die Landesregierung vor einer zweifelhaften Rechtsanwendung: Die Abtretung des Mundatwaldes sei keine Grenzberichtigung, sondern das genaue Gegenteil davon. Ein Blick auf die Landkarte nämlich zeige, dass die neue Grenze mehr als doppelt so lang wie die alte sei. Hier werde offenbar versucht, den zwischenstaatlich feststehenden Rechtsbegriff der Grenzberichtigung mit einem Inhalt zu füllen, der dem eigentlichen Sinn des Begriffs diametral entgegenstehe. ${ }^{45}$

In zahlreichen Zeitungsartikeln und Leserzuschriften wurde der Bundesregierung vorgeworfen, sie habe die Abtretung des circa 700 Hektar umfassenden deutschen Hoheitsgebietes in irreführender Weise als „Grenzberichtigung" dargestellt, während es sich in Wahrheit um eine „Grenzänderung“ handele, die nach Art. 7 Abs. 1 Satz 2 des Deutschlandvertrages bis zum Abschluss eines Friedensvertrages zurückzustellen sei. ${ }^{46}$ Eine Abtretung deutschen Hoheitsgebietes sei nach Inkrafttreten des deutsch-französischen Freundschaftsvertrages nicht mehr zeitgemäß. ${ }^{47}$ Überdies seien die in dem Abkommen vereinbarten wechselseitigen Leistungen ungleich verteilt: Die hoheitliche Abtretung des Mundatwaldes an Frankreich und die privatrechtliche Rückgewähr deutscher Grundstücke in Frankreich lägen auf verschiedenen Ebenen und würden einander nicht aufwiegen. ${ }^{48}$

Ein eigens ins Leben gerufenes „Kuratorium zur Erhaltung des Mundatwaldes“ unter Vorsitz Karl Bertzels vermutete in der diplomatisch böse verklausulierten Abmachung Lug und Trug und versuchte, Einfluss auf bundesdeutsche Parlamentarier zu nehmen, um eine Ratifizierung des Vertrages zu verhindern. ${ }^{49}$ Ein Mitglied des Kuratoriums, der sich für Heimatgeschichte interessierende Ingenieur Ludwig Schmidt aus Kaiserslautern, erstattete bei der Bundesanwaltschaft in Karlsruhe gar Anzeige wegen Staatsgefährdung. ${ }^{50}$ Die Wochenzeitung Die Zeit sprang den aufgebrachten Vaterlandsverteidigern bei und konstatierte: „In der Tat ragt der von französischen Zöllnern besetzte Mundatwald wie eine überdimensionale Nase de Gaulles in die deutschen Lande. “51

Diese Frage wurde in den vertraulichen Beratungen des Auswärtigen Ausschusses eingehend erörtert. In der 36. Sitzung des Ausschusses am 21. Juni 1967 schilderte der Vortragende Legationsrat Erster Klasse Helmut Rumpf(AA) die Entstehungsgeschichte und den Inhalt des deutsch-französischen Grenzabkommens. ${ }^{52}$ In der Aussprache wies Hermann Kopf nachdrücklich auf die vom Ausschuss bereits in der 4. Wahlperiode vertretene Auffassung

45 Siehe dazu „Zweifelhafte Rechtsanwendung in der Mundatwald-Frage? Jurist Dr. L. Ritterspacher warnt Bundes- und Landesregierung / Keine Grenzberichtigung, sondern Gegenteil“, in: Saarbrücker Landeszeitung vom 21. Dezember 1963.

46 Siehe u.a. „Unruhe um den Mundatwald: Deutsche und französische Interessen an 684 Hektar“, in: Deutsche Zeitung vom 27. Juli 1963.

47 Der Vertrag über die deutsch-französische Zusammenarbeit war am 22. Januar 1963 von Charles de Gaulle und Konrad Adenauer im Pariser Elysée-Palast unterzeichnet worden (Elysée-Vertrag). Für den Wortlaut des Vertrages siehe BGBl. 1963 II, S. 706-710.

48 Siehe den Leserbrief „Mundatwald und Staatsbewußtsein“ von Karl Bertzel, Zweibrücken, in: FAZ vom 24. September 1963.

49 Siehe Ansbert Baumann, a.a.O. (Fn. 1), S. 630.

50 Vgl. „Ein Wald als völkerrechtliches Schlachtfeld: Die Abtretung eines Quellgebiets im Kreis Bergzabern betrachten pfälzische Bürger als Staatsgefährdung“, in: SZ vom 4. April 1966.

51 Hans-Joachim Noack, a.a.O. (Fn. 9).

52 Siehe Sitzungsprotokolle, a.a.O. (Fn. 37), 36. Sitzung vom 21. Juni 1967, S. 491 f. 
hin ${ }^{53}$, dass es sich bei dem Vertrag nicht um eine Grenzberichtigung, sondern um eine Grenzänderung handele. ${ }^{54}$ Der Berichterstatter Hans-Joachim von Merkatz (CDU) griff diese Frage in der 51. Sitzung am 14. Dezember 1967 noch einmal detailliert auf. Er führte aus, dass die völkerrechtliche Literatur unter einer Grenzberichtigung (rectification) in erster Linie Begradigungen unzweckmäßig verlaufender Grenzen oder Festlegungen rechtlich ungewisser Grenzen verstehe. Die vorgesehene neue Grenze im Mundatwald sei aber schon ihrer äußeren Form nach keine Begradigung, sondern das genaue Gegenteil davon. Sie sei aber auch nicht die Ermittlung einer ungewissen Grenze. Schließlich sei die Gebietsabtretung mit ihren knapp sieben Quadratkilometern und einer Tiefe von 5,2 Kilometer auch nicht „geringen Umfangs“, so dass es für eine Grenzberichtigung an den tatbestandlichen Voraussetzungen fehle. Ein Beamter des Auswärtigen Amts hätte diese Einschätzung zwar geteilt, das Auswärtige Amt stehe im Ganzen jedoch weiterhin auf dem Standpunkt, dass nur eine Grenzberichtigung vorliege. ${ }^{55}$ Wolle man die neue Grenzziehung im Mundatwaldgebiet umschreiben, so von Merkatz weiter, müsse man sie zweifellos als Gebietsabtretung bezeichnen. ${ }^{56}$ Zudem stellte er noch einmal ausführlich die Argumente dar, die für und gegen eine Ratifizierung sprachen. ${ }^{57}$ Zur Abgabe eines abschließenden Votums sah er sich allerdings nicht imstande. ${ }^{58}$

Hermann Kopf betonte, dass ein großer Mangel des Vertrages die Ungleichwertigkeit der wechselseitigen Leistungen sei. Die hoheitliche Abtretung des Mundatwaldes an Frankreich und die privatrechtliche Rückgewähr deutscher Grundstücke in Frankreich lägen auf verschiedenen Ebenen und würden einander nicht aufwiegen. ${ }^{59}$ Zudem sei es „ein großer Schönheitsfehler", dass die privatrechtliche Abtretung von 685 Hektar des Mundatwaldes an Frankreich nur im Rahmen eines vertraulichen Schriftwechsels vereinbart worden sei, der an sich dem Parlament gar nicht zur Kenntnis kommen sollte. ${ }^{60}$

Der Abgeordnete Erich Mende (FDP) wies zum Ende der Sitzung noch darauf hin, dass der Gesetzentwurf nicht zufällig so lange bei der früheren Bundesregierung liegen geblieben sei. Zwei Ressorts hätten ihn abgelehnt, da er die Bestätigung einer Annexion und die Vorwegnahme friedensvertraglicher Regelungen beinhalte. ${ }^{61}$

(b) In den vertraulichen Beratungen des Auswärtigen Ausschusses trat überdies zutage, dass das Argument, der Mundatwald werde zur Trinkwasserversorgung der Stadt Weißenburg benötigt, nicht ganz den Tatsachen entsprach. ${ }^{62}$ Zum einen befanden sich die begehrten Quellen im vordersten südlichsten Zipfel des Mundatwaldes, so dass es nicht der Abtre-

53 Der Auswärtige Ausschuss hatte sich bereits in der 4. Wahlperiode aufgrund einer Eingabe des Bertzels in seiner 41. Sitzung am 14. November 1963 informatorisch mit dem Grenzabkommen befasst und das Auswärtige Amt ersucht, mit der französischen Regierung Verhandlungen mit dem Ziel aufzunehmen, den Mundatwald dem deutschen Staatsgebiet zu erhalten. Siehe dazu oben im Abschnitt 3.1.

54 Vgl. Sitzungsprotokolle, a.a.O. (Fn. 37), 36. Sitzung vom 21. Juni 1967, S. 493.

55 Vgl. Sitzungsprotokolle, a.a.O. (Fn. 37), 51. Sitzung vom 14. Dezember 1967, S. 686.

56 Vgl. ebenda.

57 Vgl. ebenda, S. $687 \mathrm{f}$.

58 Vgl. ebenda, S. 688.

59 Vgl. ebenda.

60 Vgl. ebenda, S. $688 \mathrm{f}$.

61 Vgl. ebenda. S. 689. Siehe dazu Fn. 18.

62 Vgl. Sitzungsprotokolle, a.a.O. (Fn. 37), 36. Sitzung vom 21. Juni 1967, S. 493 f. 
tung des gesamten Waldgebietes bedurft hätte. ${ }^{63}$ Zum anderen wären für die Wasserversorgung weitaus weniger einschneidende Maßnahmen als die Abtretung von Staatsgebiet denkbar gewesen, wie zum Beispiel die Eintragung einer Grunddienstbarkeit. ${ }^{64}$ Außerdem war es wegen des Wasserbezuges noch niemals zu Versorgungsengpässen gekommen - selbst während der beiden Weltkriege hatte Weißenburg ungehindert Wasser beziehen können.

Bei genauerem Hinsehen wurde schließlich deutlich, welches eigentliche Interesse die Franzosen am deutschen Wald zu haben schienen. Die französischen Jagdgebiete waren während des Krieges leergejagt worden. Der deutsche Wald bot indes ein gut gepflegtes Jagdrevier und war damit für die französischen Militärangehörigen unmittelbar nach dem Krieg besonders attraktiv. Die französischen Diplomaten, die für eine Abtretung des Mundatwaldes eingetreten waren und sich durchgesetzt hatten, hätten eben diese Jagdinteressen im Auge gehabt, vermuteten deshalb einige Abgeordnete wohl nicht ganz zu Unrecht. ${ }^{65}$

Über die Jahre verloren die Franzosen allerdings ihr Interesse am deutschen Wald. Die ursprünglichen Jagdinteressen spielten nicht mehr eine so große Rolle wie im Jahre 1949, als der Wald durch General Koenig unter französische Verwaltungshoheit gestellt worden war. Die französischen Jäger konnten im Gebiet des Mundatwaldes ebenso jagen wie die deutschen, da die Ausübung der Jagd durch die internationale Grenzziehung keineswegs behindert wurde. Die Stadt Weißenburg, die den Wald erhalten sollte, war auch nicht mehr in dem Maße wie früher an der Übertragung interessiert, denn die Trinkwasserversorgung war gesichert. Überdies war Waldbesitz zunehmend unrentabel geworden, und die wertvollen Bestände des Waldes waren in der Zwischenzeit offenbar abgeholzt worden. Die Stadt Weißenburg jedenfalls war durchaus geneigt, auf den Erwerb des Waldes zu verzichten, wenn ihr eine entsprechende Geldentschädigung gewährt würde. Als Summe wurde ein Betrag von sieben bis zehn Millionen DM genannt. ${ }^{66}$

(c) Der Auswärtige Ausschuss ließ die Angelegenheit zunächst liegen. Es vergingen eineinhalb Jahre, bis das deutsch-französische Grenzabkommen erneut auf die Tagesordnung gesetzt wurde. Auf der 94. Sitzung des Ausschusses am 25. Juni 1969 berichtete der Vortragende Legationsrat Erster Klasse Hans Ferdinand Linsser (AA), dass zwischen lokalen Politikern beiderseits der Grenze sehr freundschaftliche und vertrauensvolle Gespräche mit dem Ziel stattgefunden hätten, die Bestimmungen über den Grenzverlauf zu ändern. ${ }^{67}$ Bei den deutsch-französischen Konsultationsbesprechungen sei von deutscher Seite sondiert worden, ob eine Ablösung der Abtretungen durch die Einräumung eines Wassernutzungsrechts für die Stadt Weißenburg und durch die Gewährung eines finanziellen Ausgleichs zugunsten Frankreichs in Betracht gezogen werden könnte. ${ }^{68}$ Die französische Seite habe über ihre internen Konsultationen indes mitgeteilt, dass vor der Bundestagswahl im Herbst 1969 mit

63 Vgl. ebenda, S. 494 sowie 94. Sitzung vom 25. Juni 1969, S. 1365.

64 Vgl. ebenda, S. 1365.

65 Vgl. Günther Patz, Parlamentarische Kontrolle der Außenpolitik, Fallstudien zur politischen Bedeutung des Auswärtigen Ausschusses des Deutschen Bundestages, Meisenheim am Glan 1976, S. 97 ff., S. 100.

66 Vgl. Sitzungsprotokolle des Auswärtigen Ausschusses, a.a.O. (Fn. 37), 94. Sitzung vom 25. Juni 1969, S. 1365.

67 Vgl. ebenda.

68 Vgl. ebenda, S. 1366. 
einer Stellungnahme nicht zu rechnen sei. ${ }^{69}$ Als Ergebnis der Aussprache hielt Hermann Kopf fest, dass ein finanzielles Angebot an Frankreich wünschenswert sei und dass die Bundesregierung dem Auswärtigen Ausschuss in der nächsten Wahlperiode über den weiteren Verlauf der Verhandlungen berichten solle. ${ }^{70}$

In einem Schreiben an den Bundesaußenminister vom 27. Juni 1969 brachte der Ausschussvorsitzende noch einmal den ausdrücklichen Wunsch des Auswärtigen Ausschusses nach weiteren Verhandlungen mit dem Ziel einer Rückgabe des Mundatwaldes zum Ausdruck. In seinem Antwortschreiben vom 30. Juli 1969 - das dem Ausschuss auf seiner 96. Sitzung am 27. August 1969 zur Kenntnis gebracht wurde ${ }^{71}$ - teilte der Staatssekretär des Auswärtigen Amts im Auftrag des Bundesaußenministers mit, man habe in den letzten Konsultationsgesprächen konkret die Möglichkeit zur Sprache gebracht, statt der vorgesehenen Abtretung des Mundatwaldes Wassernutzungsrechte und einen eventuellen finanziellen Ausgleich einzuräumen. Die französische Seite hätte die Prüfung dieser Frage zugesagt, bei der Konsultation am 9. Mai 1969 allerdings darauf hingewiesen, dass mit einer Entscheidung über die französische Haltung dazu nicht vor der deutschen Bundestagswahl zu rechnen sei. Weiterhin habe inzwischen auch der Staatssekretär des Bundesministeriums der Finanzen auf Bitten des Auswärtigen Amts schriftlich erklärt, dass er gegen die Absicht, der französischen Regierung eine Entschädigung anzubieten, keine grundsätzlichen Bedenken erhebe. Allerdings müsse noch geklärt werden, inwieweit eine Beteiligung des Landes Rheinland-Pfalz an den Rückkaufkosten im Verhältnis zur Größe seines Besitzes am Mundatwald verlangt werden müsse. Das Auswärtige Amt bleibe um einen erfolgreichen Abschluss der Gespräche weiter bemüht. ${ }^{72}$

Eine weitere Befassung des Auswärtigen Ausschusses mit dem Abkommen vor der Bundestagswahl im Herbst 1969 erfolgte nicht. Es blieb ohne Beschlussfassung und Rücküberweisung an das Plenum im Ausschuss liegen und fiel mit dem Ablauf der 5. Wahlperiode der parlamentarischen Diskontinuität anheim. In der 6. Wahlperiode wurde das Ratifikationsgesetz nicht erneut in die parlamentarischen Beratungen eingebracht. Damit war die Ratifizierung des deutsch-französischen Grenzabkommens vom 31. Juli 1962 auf deutscher Seite gescheitert.

\section{Bemühungen der Bundesregierung um eine Neuverhandlung des Abkommens}

Durch das Verhalten des Auswärtigen Ausschusses geriet die Bundesregierung in arge Bedrängnis. Der französischen Regierung musste ein Kompensationsangebot für die vertraglich vereinbarte, aber infolge fehlender deutscher Ratifizierung des Grenzabkommens unterbliebene Abtretung des Mundatwaldes unterbreitet werden. Das Auswärtige Amt bemühte sich in der Folgezeit zunächst darum, mit der französischen Regierung ein Einvernehmen über eine Änderung des Grenzabkommens vom 31. Juli 1962 zu erzielen. Statt der endgültigen Abtretung des Mundatwaldes wurde Frankreich vorgeschlagen, eine Geldentschädigung von

69 Vgl. ebenda.

70 Vgl. ebenda, S. 1367.

71 Vgl. Sitzungsprotokolle, a.a.O. (Fn. 37), 96. Sitzung vom 27. August 1969, S. 1417.

72 Vgl. ebenda. 
einigen Millionen DM anzunehmen und der Stadt Weißenburg Wassernutzungsrechte einzuräumen. ${ }^{73}$

Die Verhandlungen mit der französischen Seite gestalteten sich allerdings schwierig. Auf eine Kleine Anfrage der CDU/CSU-Bundestagsfraktion vom 12. Dezember 1973 (BT-Drs. 7/1406) erklärte die Bundesregierung, dass konkrete Möglichkeiten, einen neuen deutschfranzösischen Grenzvertrag zur Regelung der Mundatwaldfrage auszuhandeln, nicht bestünden. ${ }^{74}$ Den Vorschlag von Neuverhandlungen über den Mundatwald lehnte die französische Regierung am 20. März 1974 ab. ${ }^{75}$

Im Zusammenhang mit einem Besuch des Bundespräsidenten Walter Scheel in Frankreich im April 1975 versuchte das Auswärtige Amt noch einmal, die französische Regierung zu Gesprächen über eine Änderung des Abkommens zu bewegen. Der deutsche Botschafter in Paris wurde deshalb an hoher Stelle im französischen Außenministerium vorstellig. Eine Antwort auf die deutschen Vorschläge ging jedoch nicht ein. ${ }^{76}$ Als das Thema auf der deutsch-französischen Direktorenkonsultation am 29. Oktober 1975 in Bonn erneut zur Sprache gebracht wurde, ließ die französische Reaktion - wie schon bei früheren Gelegenheiten - einerseits zwar ein gewisses Interesse, andererseits aber auch grundsätzliche Bedenken erkennen. ${ }^{77}$

Im Jahre 1978 kam dann noch einmal Bewegung in die Mundatwaldfrage, als auf dem deutsch-französischen Gipfel in Aachen vereinbart wurde, mögliche Entschädigungsleistungen für die elsass-lothringischen Zwangsrekrutierten des Zweiten Weltkriegs zu erörtern. ${ }^{78}$ Der damalige rheinland-pfälzische Ministerpräsident Bernhard Vogel (CDU) regte in dieser Situation an, die Mundatwaldfrage mit den Entschädigungszahlungen für die Elsässer zu koppeln und schlug ihre Einbeziehung in die Verhandlungen vor. ${ }^{79}$ Für die CDU/CSUBundestagsfraktion erklärte der Abgeordnete Alois Mertes (CDU), dass eine Zustimmung seiner Fraktion zugunsten der Zwangsverpflichteten nur dann in Frage komme, wenn diese ausdrücklich die Gegenleistung für die Rückgabe des Mundatwalds darstelle. ${ }^{80}$ Aber auch jetzt blieb Frankreich in der Mundatwaldfrage weiter hartnäckig. In dem am 31. März 1981 geschlossenen Abkommen über die Stiftung „Deutsch-französische Verantwortung“, aus der

73 Siehe dazu die Akten zur Auswärtigen Politik der Bundesrepublik Deutschland, hrsg. im Auftrag des Auswärtigen Amts vom Institut für Zeitgeschichte, München 2006, Band II: 1. Juli bis 31. Dezember 1975, Runderlass des Vortragenden Legationsrates Erster Klasse Engels vom 16. Dezember 1975, S. 1815 ff., S. 1819, Fn. 19; Siegfried Jutzi, Mundatwald und Sequesterland, in: Archiv des Völkerrechts 1986, S. 277 ff., S. 283.

74 Antwort der Bundesregierung auf die Kleine Anfrage der Abgeordneten Franz Marx, Albert Leicht, Georg Gölter und Genossen sowie der Fraktion der CDU/CSU betreffend endgültige Regelung deutsch-französischer Grenzfragen vom 3. Januar 1974, BT-Drs. 7/1494, S. 1.

75 Vgl. Heidi Dünisch, a.a.O. (Fn. 1), S. 28; Runderlass Engels, a.a.O. (Fn. 73), S. 1819, Fn. 19; Siegfried Jutzi, a.a.O. (Fn. 73), S. 283.

76 Vgl. Runderlass Engels, a.a.O. (Fn. 73), S. 1819, Fn. 19.

77 Vgl. ebenda.

78 Vgl. „Neue Hoffnung auf den Mundatwald: Koppelung der Rückgabe mit Entschädigungsleistungen an Elsässer im Gespräch“, in: Die Rheinpfalz vom 20. Juni 1979; Kleine Anfrage der Abgeordneten Ziegler, Hartard, Waldenberger, Heid und Haberer und Antwort des Chefs der rheinland-pfälzischen Staatskanzlei vom 13. April 1981, LT-Drs. 9/1385.

79 Vgl. ebenda.

80 Siehe „Entschädigung nur bei Mundatwald-Rückgabe“, in: Die Rheinpfalz vom 18. Januar 1980. 
Leistungen zur Linderung sozialer Härten an die ehemaligen Zwangsrekrutierten erbracht werden sollten, wurde der Mundatwald nicht erwähnt. ${ }^{81}$

Dies wiederum führte zu Verstimmungen auf deutscher Seite. Der Haushaltsausschuss des Bundestages ließ die Zahlung der zugesagten 250 Millionen DM am 10. Dezember 1981 unter Hinweis auf die ungelöste Mundatwaldfrage zunächst stoppen ${ }^{82}$, was bei den noch lebenden etwa 80.000 ehemaligen Zwangsrekrutierten für große Entrüstung sorgte. ${ }^{83}$ Die kaum vermittelbare Verknüpfung von Wiedergutmachungszahlungen mit einer ungelösten Territorialfrage wurde von beiden Regierungen in der Folgezeit zwar dementiert, sorgte in Frankreich aber dennoch für Misstrauen und Empörung. In der Assemblée Nationale berichtete der Straßburger Abgeordnete Emile Koehl (UDF) am 27. Oktober 1983 davon, dass der Haushaltsausschuss des Bundestages die erste Ratenzahlung an die Bedingung geknüpft habe, dass das französische Parlament einer Rückgabe des Mundatwaldes an die Bundesrepublik zustimme. ${ }^{84}$ Dieser Darstellung widersprach der französische Staatssekretär Jean Laurain zwar sogleich und betonte, dass die französische Regierung eine derartige Verknüpfung auch nie akzeptieren würde. ${ }^{85}$ Die Verhandlungsführer des Quai d'Orsay zeigten sich in den folgenden Monaten jedoch auffälligerweise zu weitaus mehr Zugeständnissen hinsichtlich des Mundatwaldes bereit als zuvor. ${ }^{86}$

\section{Einigung in der Mundatwaldfrage}

Eine Einigung wurde schließlich durch einen Notenwechsel beider Regierungen vom 10. Mai 1984 erzielt. ${ }^{87}$ Das Auswärtige Amt mied diesmal den Weg über das Parlament und zog eine Regierungsvereinbarung zwischen Deutschland und Frankreich vor. Auf dieser Grundlage sollte das noch bestehende Besatzungsrecht aufgehoben und Frankreich privatrechtlicher Eigentümer der in Art. 1 Ziff. 4 der Verordnung Nr. 212 des französischen Oberkommandierenden in Deutschland bezeichneten Gebiete des Mundatwaldes - einschließlich aller Nutzungsrechte an Forst, Wasserquellen und Jagd - werden, wobei die Ruine der Burg Guttenberg sowie private Besitzungen nicht an den französischen Staat übergehen sollten. Im Gegenzug erhielt Deutschland von Frankreich die Gebietshoheit über den Mundatwald zurück. Die britische und die amerikanische Regierung gaben diesem Plan in einer Note vom 27. August 1984 ihre Zustimmung.

Am 16. Januar 1985 wurde der deutsch-französische Notenwechsel vom 10. Mai 1984 im „Journal Officiel de la République Française“ veröffentlicht. ${ }^{88}$ Der Deutsche Bundestag

81 Vgl. Siegfried Jutzi, a.a.O. (Fn. 73), S. 284 f.

82 Dies geschah durch eine qualifizierte Sperre der Verpflichtungsermächtigung in Höhe von 250 Millionen DM, die 1983 mit 50, 1984 und 1985 mit je 100 Millionen DM fällig werden sollte. Es bestand offenbar die Absicht, die Sperre erst aufzuheben, wenn in Sachen Mundatwald ein Fortschritt erzielt würde. Vgl. Siegfried Jutzi, a.a.O. (Fn. 73), S. 284 f.

83 Vgl. Ansbert Baumann, a.a.O. (Fn. 1), S. 631.

84 Vgl. Assemblée Nationale, 7e Législature, Première Session ordinaire de 1983-1984 (45e séance), Compte rendu intégral, 3e séance, 27. Oktober 1983, S. 4668.

85 Vgl. ebenda, S. 4669.

86 Vgl. Ansbert Baumann, a.a.O. (Fn. 1), S. 631.

87 Siehe dazu Jacques Myard, L'accord du 10 mai 1984 sur le Mundat, in: Annuaire français de droit international, 31. Jg. (1985), H. 1, S. $884-892$.

88 Journal Officiel de la Republique Française, 16. Januar 1985, S. 569 - 572. 
erklärte zur Aufhebung des Besatzungsrechts ebenfalls seine Zustimmung ${ }^{89}$; das Gesetz trat am 1. Mai 1986 in Kraft. Seitdem ist der Mundatwald wieder uneingeschränkt deutsches Hoheitsgebiet, während der französische Staat Eigentümer werden sollte.

Nun blieb nur noch die Übertragung des Eigentums an den Grundstücken zu vollziehen. Im französischen Dekret vom 21. Juni 1994 zur Veröffentlichung des Abkommens zwischen der französischen und der deutschen Regierung hinsichtlich der Eigentumsrechte Frankreichs am Mundatwald, wie sie im Notenwechsel vom 10. Mai 1984 vereinbart wurden, steht, dass das von beiden Regierungen am 28. August 1990 in Nancy unterzeichnete Abkommen im „Journal Officiel“" veröffentlicht werden soll. Dieses Abkommen legte fest, wem welche Grundstücke eigentumsrechtlich gehören sollten. Die Veröffentlichung im Journal Officiel erfolgte am 28. Juni $1994 .{ }^{90}$ Damit war auch die letzte Gebietsfrage seit dem Zweiten Weltkrieg an der deutschen Westgrenze gelöst.

\section{Französische Jagdinteressen im deutschen Wald}

Auch wenn es Frankreich letztlich nicht gelang, den Mundatwald als Jagdgebiet in das französische Territorium einzuverleiben, blieb die Jagdleidenschaft der Franzosen im Mundatwald ungebrochen. Der französische Staat wurde durch die gefundene Regelung zwar privatrechtlicher Eigentümer des Mundatwaldgebietes, die Franzosen müssen sich bei der Ausübung ihres Hobbys allerdings an deutsches Recht halten. Zuletzt hatte im Jahre 2006 ein französischer Jagdausübungsberechtigter im Mundatwald einen Futterautomaten aufgestellt und mehrere nicht genehmigte Kirrungen ${ }^{91}$ angelegt und wurde daraufhin von der Kreisverwaltung Südliche Weinstraße aufgefordert, diese zu entfernen. ${ }^{92}$ Hiergegen erhob der Jäger nach erfolglosem Widerspruchsverfahren Klage und machte geltend, der Mundatwald sei kein deutsches Hoheitsgebiet, denn er sei bereits am 23. April 1949 durch die Verordnung Nr. 212 des französischen Oberkommandierenden abgetrennt und dem französischen Staatsgebiet zugeschlagen worden. Er zahle nicht nur Pacht an den französischen Staat, sondern auch die entsprechenden Steuern.

Das Verwaltungsgericht Neustadt an der Weinstraße wies die Klage ab. Die Behörde sei zu Recht auf der Grundlage der deutschen Jagdbestimmungen eingeschritten. Seit Gründung des Landes Rheinland-Pfalz am 30. April 1946 gehöre der Mundatwald zu diesem Bundesland. Durch die Verordnung 212 der französischen Militärregierung sei er zwar vorläufig unter französische Gebietshoheit gestellt worden mit der Folge, dass eine hoheitliche Betätigung deutscher Behörden nicht mehr in Betracht gekommen sei. Völkerrechtlich sei das Gebiet jedoch weiter ein Teil Deutschlands und des Landes Rheinland-Pfalz gewesen.

89 Vgl. Art. 14 des Ersten Gesetzes zur Bereinigung des Verwaltungsverfahrensrechts vom 18. Februar 1986, BGBl. 1986 I, S. 265 ff., S. 268, in dem die Aufhebung des alten Besatzungsrechts (Art. 1 Nr. 4 der Verordnung 212 des französischen Oberkommandierenden in Deutschland vom 23. April 1949) bestätigt wird.

90 Journal Officiel de la Republique Française, 28. Juni 1994, S. 9324 - 9326.

91 Lockfutter an Abschussstellen.

92 Gestützt wurde der Bescheid auf die rheinland-pfälzische Landesverordnung über die Fütterung und Kirrung von Schalenwild, wonach ungenehmigte Fütterungen und Kirrungen umgehend zu beseitigen sind. 
Im Einverständnis mit Frankreich und mit Zustimmung der britischen und amerikanischen Regierungen sei die Verordnung Nr. 212 mit Wirkung zum 1. Mai 1986 aufgehoben worden. Seither unterliege der Mundatwald wieder der uneingeschränkten deutschen Hoheitsgewalt. Im Gegenzug sei die französische Republik nach deutschem Recht als Eigentümerin bestimmter Bereiche des Mundatwaldes in das Grundbuch eingetragen worden. Hoheitsrechte, insbesondere im Bereich der Jagd, seien dem französischen Staat aber nicht verblieben. ${ }^{93}$

\section{Resümee: Der Fall des Mundatwaldes als Lernprozess der Regierung}

Im Falle des Mundatwald-Abkommens hat der Auswärtige Ausschuss des Bundestages gezeigt, dass er durchaus in der Lage ist, das Zustandekommen völkerrechtlicher Verträge der Bundesregierung, mit denen er nicht einverstanden ist, zu verhindern. Erstmals stellte sich der Ausschuss fraktionsübergreifend gegen ein von der Bundesregierung ausgehandeltes völkerrechtliches Abkommen. Wegen grundsätzlicher Bedenken der Abgeordneten kam ein abschließendes Votum des Auswärtigen Ausschusses zur Vorlage des Ratifikationsgesetzes zum deutsch-französischen Grenzabkommen vom 31. Juli 1962 an das Plenum des Bundestages nicht zustande. Der Gesetzentwurf blieb unerledigt liegen und fiel mit Ablauf der 5. Wahlperiode der parlamentarischen Diskontinuität anheim. In der darauffolgenden Wahlperiode wurde das Ratifikationsgesetz nicht erneut in die parlamentarischen Beratungen eingebracht. Damit war das deutsch-französische Grenzabkommen gescheitert. Durch schlichte Nicht-Beschlussfassung hatte der Auswärtige Ausschuss das Inkrafttreten des Abkommens verhindert.

Der hier geschilderte Vorgang war nicht nur der erste, sondern der bislang einzige Fall, in dem die Zustimmung zu einem von der Bundesregierung ausgehandelten völkerrechtlichen Vertrag vom Bundestag verweigert wurde. ${ }^{94}$ Eine weitere Verweigerung parlamentarischer Zustimmung bei der Ratifizierung völkerrechtlicher Verträge gem. Art. 59 Abs. 2 Satz 1 GG ist in der Staatspraxis der Bundesrepublik Deutschland bislang nicht bekannt geworden. ${ }^{95}$ Die Bundesregierung war seither klug genug, den Bundestag beziehungsweise die Mitglieder seines Auswärtigen Ausschusses während laufender Vertragsverhandlungen frühzeitig zu informieren und zu unterrichten, etwaigen Bedenken der Abgeordneten Rechnung zu tragen und sich so der Zustimmung des Parlaments im Ganzen zu versichern.

93 Verwaltungsgericht Neustadt, Urteil vom 27. August 2007, Aktenzeichen 4 K 596/07 NW. Siehe auch den Beschluss es OVG Rheinland-Pfalz vom 13. August 2008, Aktenzeichen 8 A 11351/07 OVG.

94 Siehe auch Volker Pilz, Der Auswärtige Ausschuss des Deutschen Bundestages und die Mitwirkung des Parlaments an der auswärtigen und internationalen Politik, Berlin 2008, S. 69, Fn. 31.

95 Vgl. Dietrich Rauschning, Bonner Kommentar zum Grundgesetz, Art. 59 Rn. 93, Loseblatt, 143. Aktualisierung, Dezember 2009. 